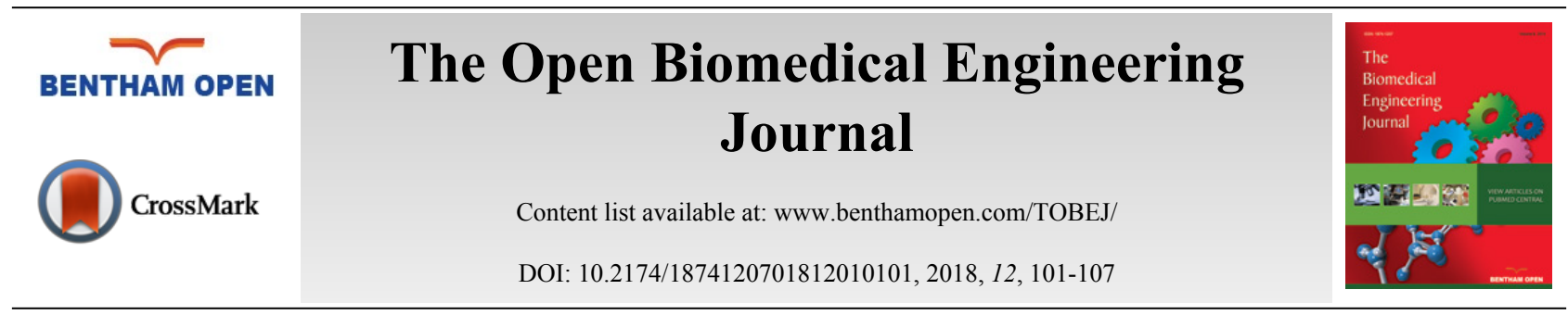

RESEARCH ARTICLE

\title{
Load Cells Application for Developing Weight-Bearing Detection via Wireless Connection
}

\author{
Tossaphon Jaysrichai*
}

Division of Physical Therapy, Faculty of Physical Therapy, Srinakharinwirot University, Nakhon Nayok, Thailand

Received: August 01, 2018

Revised: November 12, 2018

Accepted: November 15, 2018

\section{Abstract:}

Objective:

To focus on a device development for measuring the weight-bearing on feet while simultaneously reporting data on smartphone usage. Secondly, the output data from this device are tested for the accuracy.

\section{Methods:}

Researcher used eight pieces of $50 \mathrm{~kg}$ Body Load Cell Weighing Sensor Resistance strain Half-bridge Original (four pieces per foot), two pieces of HX711 amplifier, MCS-51 AT89 C51 microcontroller and HC-05-Bluetooth to develop the device. This device is called the Wireless Weight-Shifting Detector (WWSD) which is used to measure weight-bearing on feet by being attached to sandals. The output data are simultaneously reported on smartphone via line-graphs [load (kg) per sampling data] and numeric by FootpressV1 application. The device was tested using accurate measuring data with standard weights and calculated in percentage of accuracy (\%). After that, WWSD was used to compare the weight-bearing measurement during standing with forceplate (AMTI; OR6-7 Platform).

\section{Results:}

The percentage of accuracy of WWSD was more than $97 \%$ and the error values from the measuring standard weights ranged under $\pm 0.23 \mathrm{~kg}$. The accuracy of measurement was more than $95 \%$ when compared with the force plate.

\section{Conclusion:}

WWSD can report simultaneously the weight-bearing on feet with rather accurate data on smartphone. It may be helpful in assisting physical therapists while training patients in clinic.

Keywords: Weight-Bearing, Assistive Technology, Wireless Technology, Load Cell, Ambulatory Device, Physical therapists.

\section{INTRODUCTION}

The weight-bearing on feet is a method of physical therapy assessment. An information about the weight-bearing between the left-side and the right-side refers to the effectiveness of balance system and also shows a good alignment and normal functioning of the back and the legs [1 - 3]. Thus, the weight-bearing on feet is used for the physical therapy assessment on both neurological $[4,5]$ and orthopedics assessments [6 - 8].

The clinical assessment of weight-bearing on feet uses typically the scales (spring scale or digital scale), however, there are some limitations. The spring scale has a problem with the reading data. The digital scale reports only a value per measurement, thus, it cannot continuously report the output data. Finally, the user must read the output only on the screen, therefore, it is very difficult to achieve feedback information for training patients.

\footnotetext{
* Address correspondence to this author at the Division of Physical Therapy, Faculty of Physical Therapy, Srinakharinwirot University, Nakhon
} Nayok, Thailand, Tel. +669-4593-5464; E-mail:tossaphon@g.swu.ac.th 
In this study, the interested researcher developed a new device to use for weight-bearing on feet. The $50 \mathrm{~kg}$ Body Load Cell Weighing Sensor Resistance Strain Half-bridge DIY (silver) (ThaiEasyElec.com (Venus Supply Company Limited), Bangkok, BKK, Thailand) that is a load cell sensor in the digital scale is applied to developing a device for detecting the weight-bearing. The purpose was the development of weight-bearing detection device. Moreover, the device could send the data to smartphone or tablet in real-time. After that, the researcher brought the device to test for an accurate data reading.

\section{MATERIALS AND METHODS}

\subsection{Wireless Weight-Shifting Detector}

Eight of $50 \mathrm{~kg}$ Body Load Cell Weighing Sensor Resistance Strain Half-bridge DIY (silver) (ThaiEasyElec.com (Venus Supply Company Limited), Bangkok, BKK, Thailand) were selected to compound with two HX711 Load cell

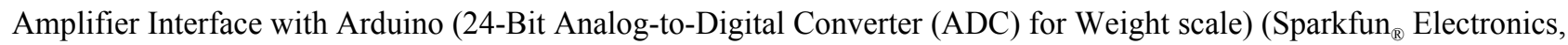
Colorado, CO. USA), an MCS-51 Microcontroller board Atmel AT89 C51 (ATMEL Corporation, Colorado, CO. USA) and Module HC-05-Bluetooth to Serial Port (ThaiEasyElec.com (Venus Supply Company Limited), Bangkok, BKK, Thailand). All of them were built in two special-designed acrylic plates for each foot. The Lithium battery in a power bank was used as the power supplied system via micro-USB port. They were brought to develop a weight-bearing detection device (Fig. 1). Total prices for the material of development were around 120 US dollars. The researcher designed this device as pads to attach under the sandals. Sandals and the device were attached together using Velcro tape. This device could record the weight-bearing data as $4 \mathrm{~Hz}$ sampling rate data (4 data per a second). The data of weight-bearing on each foot were measured separately into the front-part and the hind-part and also added for the weight-bearing data on each foot. The weight-bearing data were sent to the smartphone via Bluetooth and were reported in the numeric data and the line-graph by FootpressV1 application. This application on smartphone, which worked on the Android system, was created on the MIT App inventor (Free program; Massachusetts Institute of Technology $<$ MIT $>$, Cambridge, UK). This developed device was called Wireless Weight-Shifting Detector $<$ WWSD $>$.

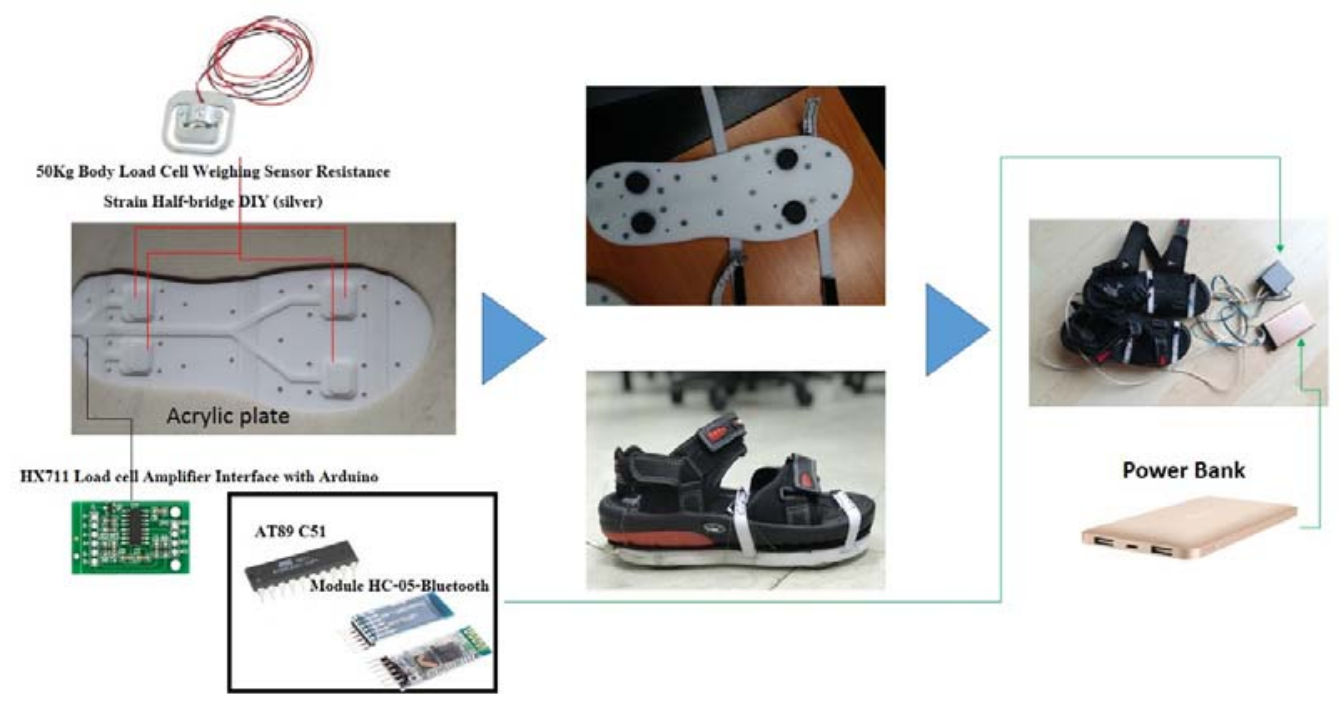

Fig. (1). Shows the components of Wireless Weight-Shifting Detector (WWSD).

\subsection{FootpressV1 Application}

Footpress Version 1 application is the software on Android smartphone that operates on the Android version 7.0. It is used for reporting data of weight-bearing on smartphone by the numeric data and the line-graph. When opening the application on smartphone, it must input the file-name data. Afterwards, the user checks the connection between the WWSD and the smartphone. If the device already links to the smartphone, there is a "beep" sound or vibration and the data are plotted into the line-graphs. The "Tare" command is used to set "zero" data. It is used to calibrate data before using the device. There are four graphs on the screen. Four line-graphs were the relationships between the weightbearing (in kilograms) and no. of data. The first graph is the red line-graph that reports the values of weight-bearing 
(in kilograms) on the front-part of the left foot via sampling data. Also, it is reported into numeric data under the "L Front" block. The second is the green line-graph that reports the values of weight-bearing (in kilograms) on the hindpart of the left foot. The dark blue line-graph represents the values at the front-part of the right foot and the pink linegraph represents the values at the hind-part of the right foot. The numeric data are reported under the block of "L Rear", "R Front" and "R Rear", respectively. The data of "L Front" and "L Rear" are summed into the weight-bearing of the left foot and the data of "R Front" and "R Rear" are summed into the weight-bearing of right foot. Moreover, this application can set the targets of weight-bearing on each foot, it will be an alarm with a sound or a vibration when the target is reached (Fig. 2). The measuring data were recorded into the smartphone by .txt file type.

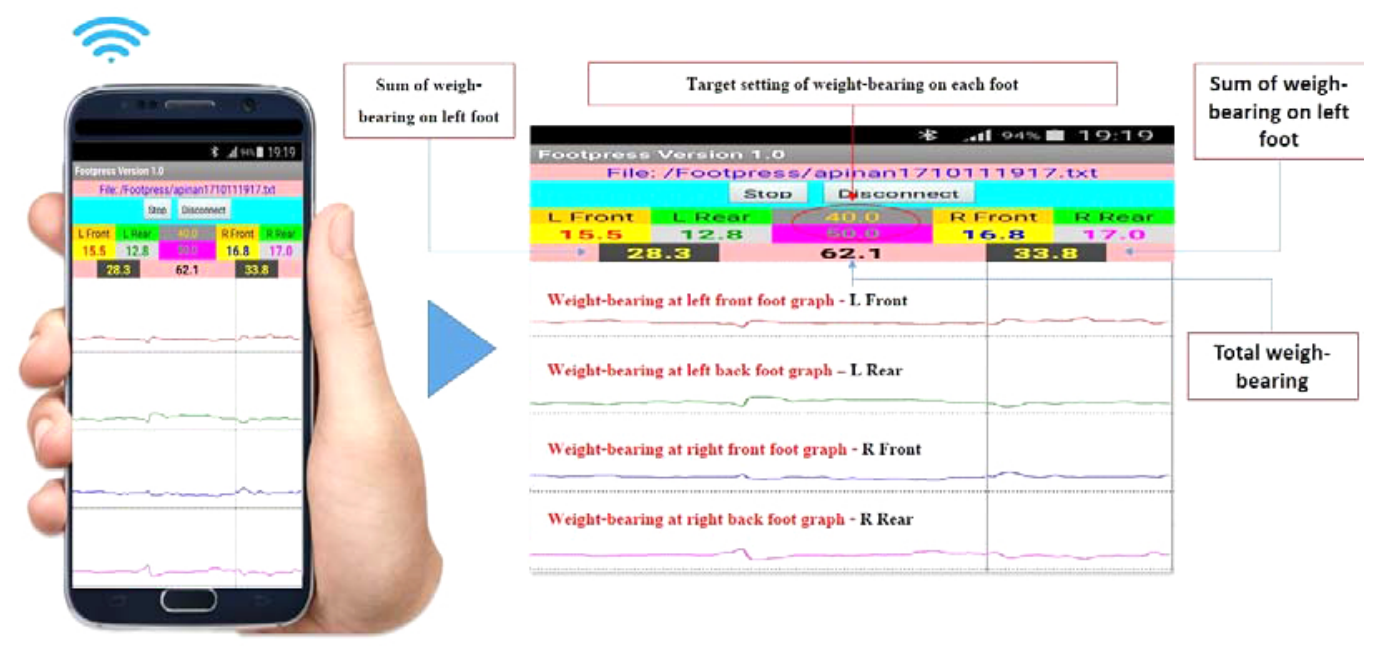

Fig. (2). Shows the layout of footpressV1 application. On the screen, There are the detail of current filename, the "Stop" and "Disconnect" buttons, the numeric outputs of weight-bearing on front-part of left foot (L Front) and hind-part of left foot (L Rear) and front-part of right foot (R Front) and hind-part of right foot (R Rear), the sum of weight-bearing on left foot, the sum of weightbearing on right foot, the total weight-bearing and the target setting of weight-bearing on each foot. There are the four line-graphs that represent the relationship between weight-bearing and the sampling data (4 times per second). They compose with the weightbearing on front-part of left foot, hind-part of left foot, front-part of right foot, and hind-part of right foot.

The cooperation between the Footpress Version 1 application and WWSD is shown on the flowchart in Fig. (3).

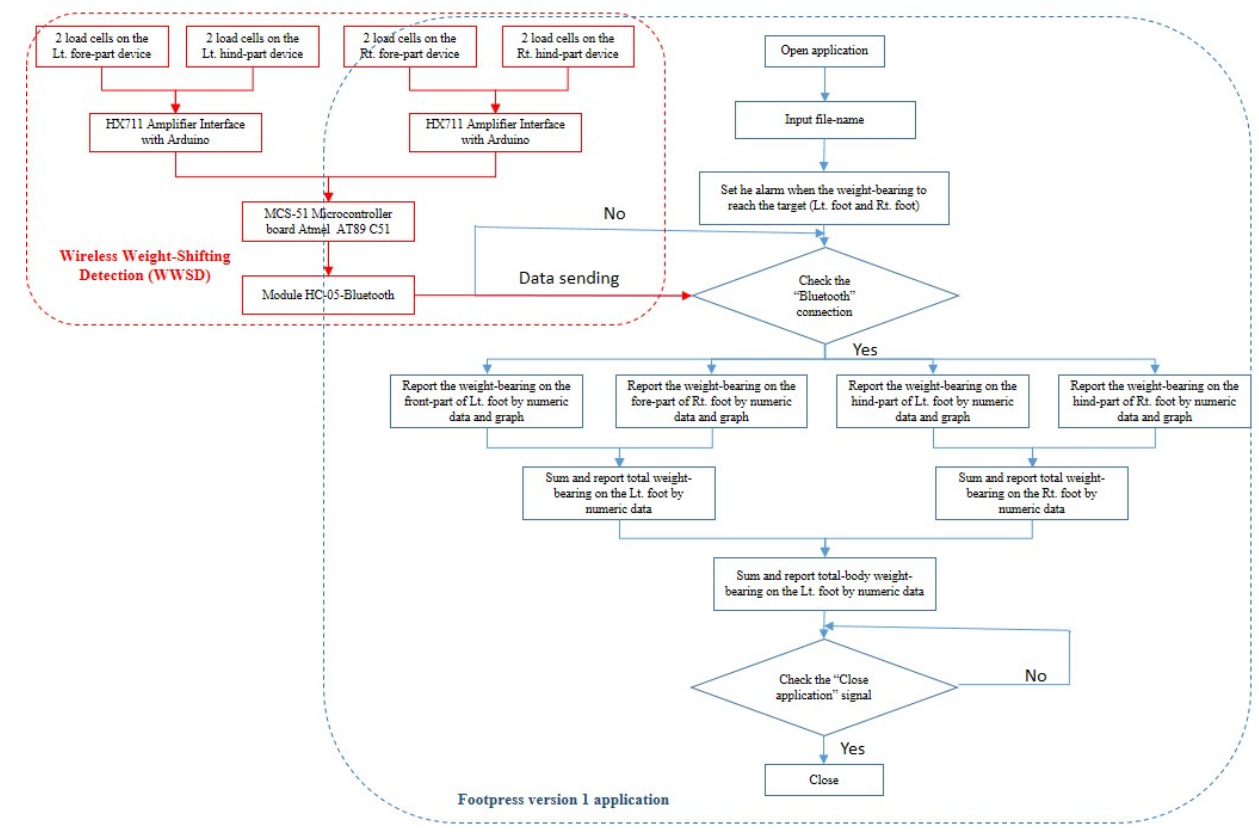

Fig. (3). Shows the flowchart of developed WWSD and Footpress application. 


\subsection{The Testing Protocol}

The researcher brought the WWSD to test the accuracy of measurement. Firstly, the standard weight size 1, 2.5, 5, $7.5,10,12.5,15,17.5,20,22.5,25$, and 27.5 kilograms were used to test the measurement of the left and right parts of WWSD. Each weight size was tested 3 times (Fig. 4). The researcher recorded the data after putting the weights on the WWSD for 10 seconds. The output data were an average of 3 seconds (12 output data). An average value from WWSD measurement was compared to the corrected value of weight. Then, the researcher calculated the percentage of accuracy $(\%)$ and the percentage of error $(\%)$.
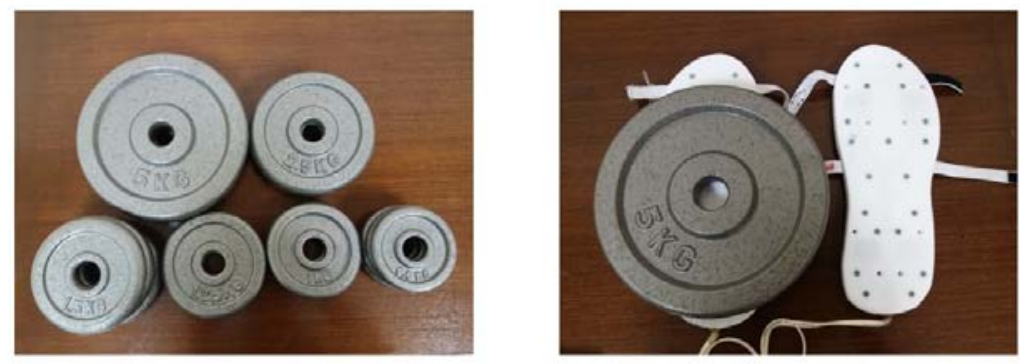

Fig. (4). Shows the accuracy test of WWSD with standard weights.

$$
\begin{gathered}
\text { Percentage of accuracy }(\% \text { Accuracy })=100 \text { - \% Error } \\
\text { Relative error }=\left|\frac{X_{\text {measure }}-X_{\text {true }}}{\boldsymbol{X}_{\text {true }}}\right| \\
\text { \%Error = Relative error x } 100 \\
X_{\text {measure }} \text { is the measuring output value } \\
X_{\text {true }} \text { is the true value }
\end{gathered}
$$

Secondly, the researcher brought the WWSD to compare the weight-bearing measurement with forceplate (AMTI; OR6-7 Platform) (Interface Advanced Force Measurement, Berkshire, England). The researcher needed to measure errors of WWSD's outputs from the standard device's output. The researcher measured the WWSD's weight by forceplate. After that, the researcher wore the WWSD and also stood on the forceplate (Fig. 5). The weight-bearing data from forceplate were minuse with the WWSD's weight and compared to the outputs from the WWSD.

Thirdly, the researchers brought the WWSD to measure the weight-bearing on standing. Thirteen volunteers participated in this test. They were asked to wear the WWSD and to stand on two digital scales. Each foot placed on each center of the digital scale. The weight-bearing outputs on each digital scale were minuse with the WWSD's weight and sandals were compared to the outputs from WWSD. All subjects were tested in a standing position three times. This protocol was approved by the Ethics Committee Board from Faculty of Physical therapy (PTPT2017-021).
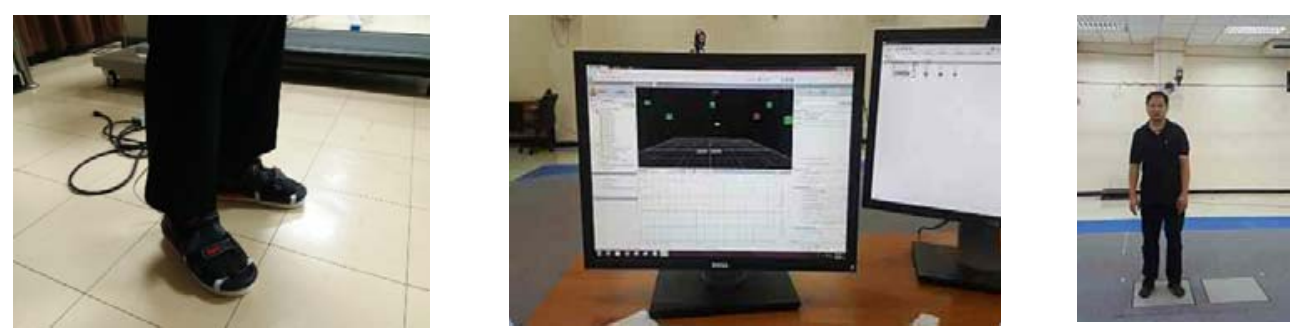

Fig. (5). Shows the accuracy test of WWSD with the forceplate (AMTI; OR6-7 Platform). 


\section{RESULTS}

For the accuracy test with the standard weights, these results reported the differences of WWSD's outputs from the standard weights. Also, the researcher calculated the different values of percentage of accuracy (\%) (Fig. 6). For the accuracy test with forceplate, they found a close relationship of weight-bearing outputs between the forceplate and WWSD (Fig. 7). When brought the WWSD to test weight-bearing on the feet from thirteen participants, the results revealed the average different values between the WWSD's output data and digital scales to be $0.62 \mathrm{~kg}$ on the left foot and $0.89 \mathrm{~kg}$ on the right foot (Fig. 8).

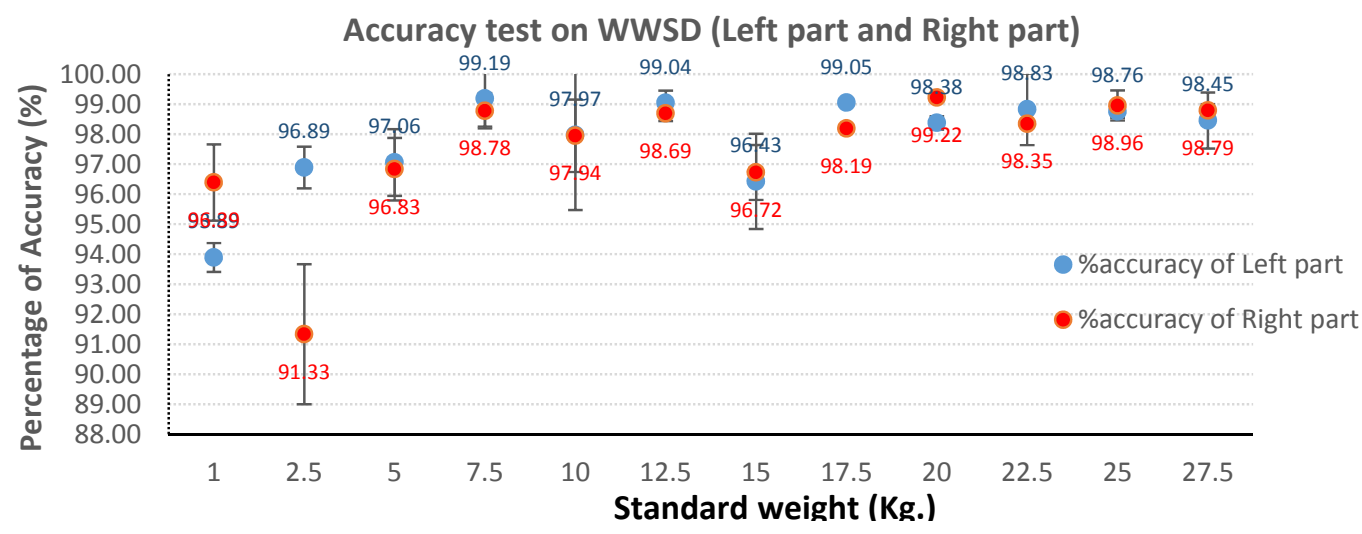

Fig. (6). Shows the comparison of accuracy in terms of percentage (\%) between the WWSD's output data from left part (Red) and right part (Blue) and the standard weights.

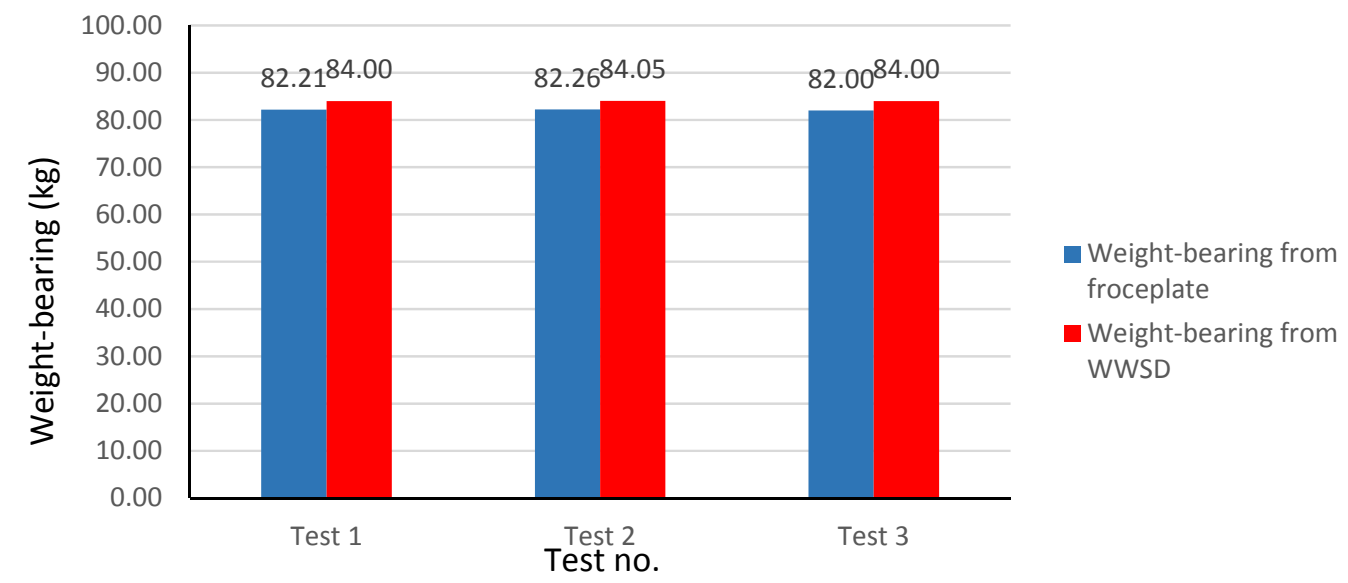

Fig. (7). Shows the comparison (in kilograms) between the WWSD's output data and the forceplate (AMTI; OR6-7 Platform).

\section{DISCUSSION}

The purpose of this study was to measure continuously the quantitative data of weight-bearing on feet. Thus, the researcher developed the WWSD that was based on load cells, microcontroller circuits, and the wireless system (Bluetooth). Load cells (50 kg Body Load Cell Weighing Sensor Resistance Strain Half-bridge DIY (silver)) are the component that is used in the digital scale. The digital scale uses four load cells, which are placed on each edge, to calculate a total weight. The digital scale had limitations because it reported only a certain value per measurement. It was not an appropriate tool for training patients when the therapists needed to measure the controlled weight-bearing on the feet of the patients. The continuous data, for example the spring scale, are important for feedback. However, the spring scale has limitations for reading data. The researcher applied the load cells with HX711 Load cell Amplifier Interface with Arduino (24-Bit Analog-to-Digital Converter $<$ ADC $>$ for Weight scale) and an MCS-51 Microcontroller 
board Atmel AT89 C51, a battery supplied to transform the electrical voltage outputs to weight-bearing data. The researcher separated two couples of load cells into a couple for the front-part and a couple for the hind-part. It could report the output data for the weight-bearing on the front-part of the foot and the weight-bearing on the hind-part of foot (four load cells per foot). Each base of WWSD made from two acrylic plates to place under the sandals because the WWSD need a stable base for using measurement with load cells. The researcher developed the WWSD in terms of the plates for using under the sandals because it was suitable for different foot sizes of users. There were four load cells and an HX711 Load cell Amplifier Interface with Arduino and cables inside each acrylic plate (Fig. 1). Then, the output signals of weight-bearing on feet were sent to an MCS-51 Microcontroller board Atmel AT89 C51 and Module HC-05Bluetooth by cables and transformed the output data into the wireless signals. The researcher created the application on smartphone to receive and to display the output data. The weight-bearing output data were reported instantly on linegraphs and numeric data. There were four line-graphs that represented the output data from the front-part of the left foot, the hind-part of the left foot, the front-part of the right foot, and the hind-part of the right foot. This application could set the target of weight-bearing on each foot and also has an alarm when achieving the target via sound or vibration. So, it can help the therapists to train the weight-bearing for their patients.

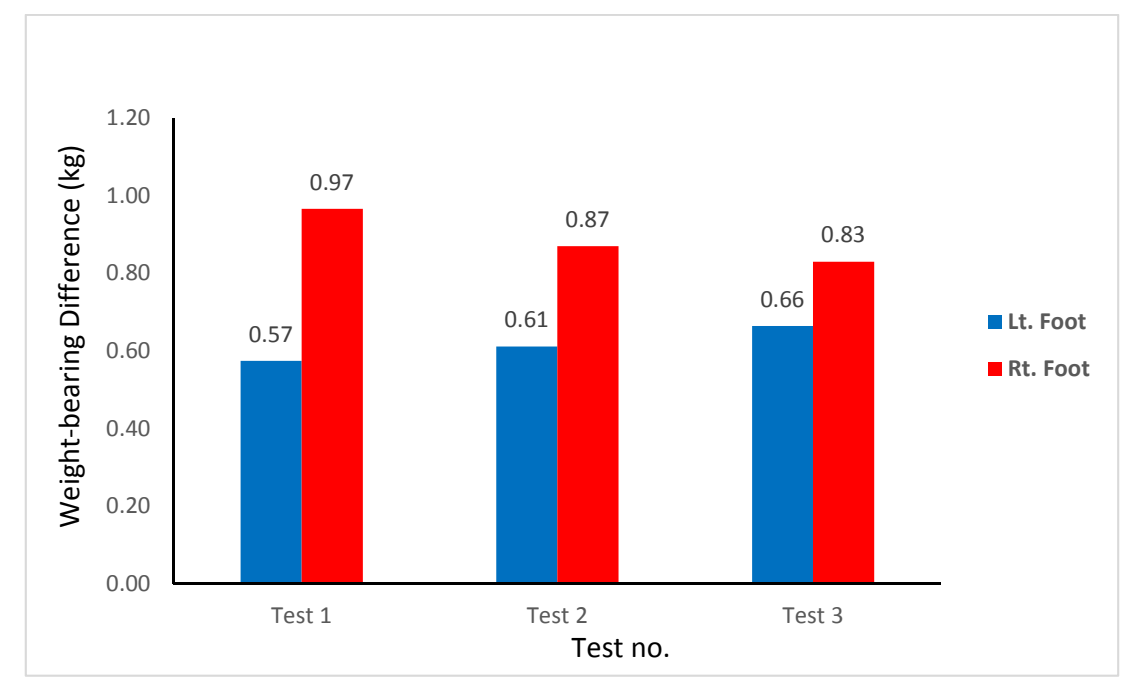

Fig. (8). Shows the comparison of weight-bearing difference (in kilograms) between the left \& right WWSD's output data and the digital scales.

For the accuracy test with the standard weights, a high percentage of accuracy was found when compared with 12 standard weights. The left side of WWSD found the percentage of accuracy to be 97.83 percent and to be $97.52 \%$ on the right side of WWSD. For the comparison between weight-bearing measurement and forceplate, the average different value was found to be $1.86 \mathrm{~kg}$. and the percentage of accuracy to be $97.74 \%$. These results revealed a good accuracy for WWSD. Then, WWSD introduced to test the weight-bearing measurement on thirteen participants while standing. This revealed that it could be applied to measuring weight-bearing.

The WWSD still had some limitations. It was quite a height from the floor because the total height of WWSD was 1.47 centimeter $(\mathrm{cm})$ and it added around $1 \mathrm{~cm}$. to the height of the sandals. Subjects may have felt an instability while wearing it. The WWSD cannot be flexible, thus, it is not suitable to record data during walking. The WWSD was 1.5 kg. per side, thus, it was quite heavy when walking with WWSD. The HX711 was used in the WWSD and had restrictions as the gains from channel A and B were different. Channel A had gains of 128 and Channel B had gains of 32. The researcher brought the outputs from channel B to multiply by four, thus, the outputs from the hind-part were unstable data.

This device also had a benefit for the clinical assessment or training of weight-bearing on feet. It could apply for evaluating weight-bearing on different standing positions i.e. normal standing, step standing. The WWSD reported immediately the weight-bearing data on the smart phone via Bluetooth system, so that, it helped to give feedback data for the patients. For further study, the researcher will bring the WWSD to test on the weight-bearing on the foot with ankle instability. 


\section{CONCLUSION}

In conclusion, the WWSD, which is developed in this study, can help the therapists to train or assess their patients on weight-bearing on feet. It was found to be accurate when compared with the outputs. It may be applied to assess and to train the weight-bearing on feet for neurologic or orthopedic patients.

\section{ETHICS APPROVAL AND CONSENT TO PARTICIPATE}

Not applicable.

\section{HUMAN AND ANIMAL RIGHTS}

No Animals/Humans were used for studies that are base of this research.

\section{CONSENT FOR PUBLICATION}

Not applicable.

\section{CONFLICT OF INTEREST}

The authors declare no conflict of interest, financial or otherwise.

\section{ACKNOWLEDGEMENTS}

I would like to sincerely thank the Srinakharinwirot University, Thailand for the grant support. I also thank the Faculty of Physical Therapy for forceplate (AMTI; OR6-7 Platform) at Srinakharinwirot University, Thailand that allowed me to use the facilities for this study.

\section{REFERENCES}

[1] D. de Kam, J.F. Kamphuis, V. Weerdesteyn, and A.C.H. Geurts, "The effect of weight-bearing asymmetry on dynamic postural stability in people with chronic stroke", Gait Posture, vol. 53, pp. 5-10, 2017. [http://dx.doi.org/10.1016/j.gaitpost.2016.12.019] [PMID: 28061401]

[2] R. Dickstein, M. Nissan, T. Pillar, and D. Scheer, "Foot-ground pressure pattern of standing hemiplegic patients. Major characteristics and patterns of improvement", Phys. Ther., vol. 64, no. 1, pp. 19-23, 1984. [http://dx.doi.org/10.1093/ptj/64.1.19] [PMID: 6691049]

[3] S. Döbele, C. Deininger, G.H. Sandmann, A. Schmitt, T. Freude, U. Stöckle, and M. Lucke, "New method for monitoring partial weight bearing (PWB) of outpatients with an independent insole sensor system", Acta Chir. Orthop. Traumatol. Cech., vol. 83, no. 2, pp. 88-93, 2016

[PMID: 27167422]

[4] M. Galli, V. Cimolin, M. Pau, B. Leban, R. Brunner, G. Albertini, and G. Albertini, "Foot pressure distribution in children with cerebral palsy while standing", Res. Dev. Disabil., vol. 41-42, pp. 52-57, 2015. [http://dx.doi.org/10.1016/j.ridd.2015.05.006] [PMID: 26057837]

[5] H.L. Hurkmans, J.B. Bussmann, E. Benda, J.A. Verhaar, and H.J. Stam, "Effectiveness of audio feedback for partial weight-bearing in and outside the hospital: A randomized controlled trial", Arch. Phys. Med. Rehabil., vol. 93, no. 4, pp. 565-570, 2012. [http://dx.doi.org/10.1016/j.apmr.2011.11.019] [PMID: 22325684]

[6] M.T. Manley, and E. Solomon, "The clinical assessment of the normal and abnormal foot during locomotion", Prosthet. Orthot. Int., vol. 3, no. 2, pp. 103-110, 1979 . [PMID: 503791]

[7] Z. Pataky, D. De León Rodriguez, A. Golay, M. Assal, J.P. Assal, and C.A. Hauert, "Biofeedback training for partial weight bearing in patients after total hip arthroplasty", Arch. Phys. Med. Rehabil., vol. 90, no. 8, pp. 1435-1438, 2009. [http://dx.doi.org/10.1016/j.apmr.2009.02.011] [PMID: 19651281]

[8] S Pichaiyongwongdee, J Khamdej, and C Suwanasri, "Weight distribution during standing in thai subjects aged 11-80 years", Thai. J. Phy. Ther., vol. 17, no. 2, pp. 29-43, 1995.

(C) 2018 Tossaphon Jaysrichai.

This is an open access article distributed under the terms of the Creative Commons Attribution 4.0 International Public License (CC-BY 4.0), a copy of which is available at: https://creativecommons.org/licenses/by/4.0/legalcode. This license permits unrestricted use, distribution, and reproduction in any medium, provided the original author and source are credited. 